\title{
Late-onset Lennox-Gastaut syndrome as a phenotype of 15q11.1q13.3 duplication
}

\author{
João Rocha ${ }^{1}$, Cátia Guerra ${ }^{2}$, Renata Oliveira ${ }^{3}$, Sofia Dória ${ }^{4}$, \\ Ricardo Rego ${ }^{5}$, Maria José Rosas ${ }^{6}$ \\ ${ }^{1}$ Neurology Department of Hospital de Braga \\ 2 Psychiatry Department of Hospital de São João, Porto \\ 3 Department of Medical Genetics of Hospital Pediátrico Carmona da Mota, Coimbra \\ ${ }^{4}$ Faculty of Medicine, University of Porto/Human Genetics Department, Hospital São \\ João, Porto \\ ${ }^{5}$ Neurophysiology Department of Hospital de São João, Porto \\ ${ }^{6}$ Neurology Department of Hospital de São João, Porto, Portugal
}

Received December 15, 2011; Accepted March 14, 2012

ABSTRACT - The clinical symptoms associated with chromosome $15 q$ duplication syndrome manifest through a heterogeneous group of symptoms characterised by hypotonia, delay in motor skills and language development, cognitive and learning disabilities, autism spectrum disorder and refractory epilepsy. The late development of Lennox-Gastaut syndrome in patients with 15q11q13 duplication is a possibility that physicians should be aware of. We report the case of a 27-year-old man with a neurodevelopmental syndrome due to a $15 q$ duplication, with intellectual disability, psychiatric disturbances, and an epileptic phenotype diagnosed as late-onset LennoxGastaut syndrome.

Key words: $15 q$ duplication, Lennox-Gastaut syndrome

The appropriate regulation of imprinted gene expression across chromosome $15 q 11 q 13$ has important implications for human disease and many rearrangements may occur in this imprinted segment due to the instability of this region. Three different neurodevelopmental disorders result from aberrant expression of imprinted genes in this region: Prader-Willi syndrome and Angelman syndrome (AS), which are mainly due to deletions, and $15 q$ duplication syndrome. Each of these disorders occurs at an estimated frequency of 1/15,000-1/30,000 live births (Battaglia, 2005).
The syndrome of $15 q$ duplication may be clinically suspected and result from either interstitial duplication of $15 q 11 q 13$ or an extra isodicentric chromosome 15 (idic15) (Battaglia, 2008).

These individuals commonly present with hypotonia, delay in motor skills and language development, cognitive and learning disabilities, refractory epilepsy, and, rarely, characteristic facial features. Affected individuals may meet the diagnostic criteria for autism (Cook et al., 1998; Chamberlain and Lalande, 2010) or may have a distinctive "autistic-like" trait such as 
in idic15 patients (Battaglia et al., 2010). Lennox-Gastaut syndrome (LGS) has rarely been associated with these chromosomopathies (Battaglia, 2008; Orrico et al., 2009).

Lennox-Gastaut syndrome is characterised by the triad of: multiple generalised seizure types, including atypical absences, atonic and tonic seizures; cognitive regression/intellectual disability; and generalised slow spike-and-wave complexes during wakefulness and generalised fast paroxysmal activity during sleep (Campos-Castelló, 2004).

We report a case of a patient with a dup15(q11.1q13.3) karyotype with intractable epilepsy and cognitive and psychiatric disturbances, who was diagnosed with lateonset Lennox-Gastaut syndrome.

\section{Case Study}

A 27-year-old male was followed in the Neurology Department as an outpatient due to refractory epilepsy. He had previously visited a neuropaediatric unit since he was 6 , where his karyotype was found to be $46 \mathrm{XY}$, dup15(q14q15). His family history was negative for neurological or psychiatric disorders and no genetic anomaly was found based on his parents' karyotype.

He presented with some dysmorphic features including low posterior hairline, a long face with a depressed glabella, bilateral ptosis of the eyelid, prominent and wide nasal bridge with broad nasal tip, short columella and philtrum, and broad chin. His ears showed slight posterior rotation. He had also unilateral strabismus (left exotropia) (figure 1).

He was the second child born of healthy, young and non-consanguineous parents. After an unremarkable pregnancy, there was a period of prolonged labour after 40 weeks of gestation, having required an emergency rescue intervention followed by 3-day follow-up in an ICU. Apgar scores were not available on file. Somatometric parameters were normal without dysmorphic features reported at that time.

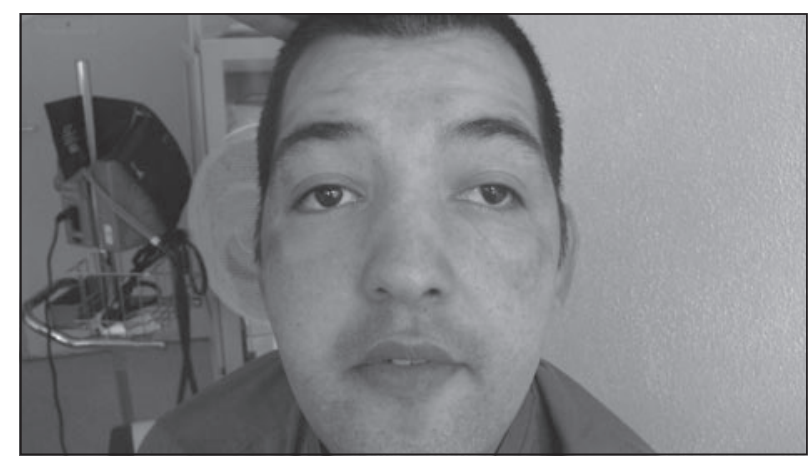

Figure 1. Photo of the patient.
Very soon afterwards, psychomotor retardation became obvious. When awake, he was poorly reactive to visual and auditory stimuli. Poor weight gain and hypotonia were observed from the first months. Smiling was achieved at the age of three months, he sat at the age of eight months, and he walked unsupported at 18 months, with his trunk leaning to the left.

Speech development was significantly delayed but he said his first words at the age of 15 months. Intellectual disability seems to have progressed throughout his life with growing learning difficulties, having never acquired reading or writing skills. Expressive communication and comprehension difficulties are growing and concomitantly he shows poor motor coordination, with aggravating ambulatory difficulties.

Concerning his behaviour pattern, he had acceptable social interaction and autistic traits were never identified. Problematic behavioural changes started at age 17 with abnormal anxiety and irritability. At age 20, he exhibited psychotic symptoms. He had episodes of severe agitation with aggressiveness towards his parents and self-injuring behaviour. These behaviour alterations were difficult to control with psychotropic medications. Anti-psychotic drugs, such as melperone and cyamemazine, had been used to control symptoms.

His seizure history started when he was 10 years old, with two consecutive, secondary generalised, right versive seizures. His interictal electroencephalogram (EEG) and brain CT scan were reported to be normal and no treatment was initiated. Three years later, he presented with another focal left tonic-clonic seizure with secondary generalisation. Again, his interictal EEG was normal. He was started with carbamazepine treatment with excellent response.

At age 24, he was admitted to hospital for focal non-convulsive status epilepticus. Clinically, he was stuporous and had subtle right and upward eye and head movements. Simultaneous EEG showed repetitive left or right fronto-temporal ictal patterns. This episode of status was controlled with carbamazepine, levetiracetam, lamotrigine and clonazepam.

At that time, cerebral MRI was performed which was normal. Since then, his epilepsy worsened with an increasing frequency of seizures, despite multiple efforts of antiepileptic optimisation (several combinations with the previously reported drugs and also sodium valproate plus clobazam). Seizure types still included right versive seizures with secondary generalisation, but over the last year he started having clusters of tonic and atonic seizures. He now has at least one tonic seizure per week and several head drop episodes per day. In his most recent EEG, we recorded brief axial tonic seizures in sleep, as well as generalised 
epileptiform activity, including generalised slow spikewave complexes during drowsiness and generalised fast paroxysmal activity in sleep (figure 2).

In 2011, due to a lack of reports concerning his chromosome structural anomaly as well as the improvement of cytogenetics techniques, another karyotype was performed to better characterise the duplication. The karyotype confirmed a duplication on chromosome 15 , but involving the $\mathrm{q} 11.1 \mathrm{q} 13.3$ region rather than the q14q15 region previously described.

\section{Discussion}

Patients with duplication of chromosome 15q11q13 usually present with hypotonia, delay in motor skills and language development, cognitive and learning disabilities, and epilepsy. Affected individuals usually meet the diagnostic criteria for autism. In fact, one study of autistic children identified $15 q$ aberrations as the single most common cause and estimated the prevalence of $15 q$ duplication in autism at $1-3 \%$ (Cook et al., 1998).

Our patient presented with the developmental anomalies associated with $15 q$ duplication, although his behavioural disorder did not fit the profile of autism or autism spectrum disorder (ASD). It has been reported that the ASD phenotype does not appear to be fully penetrant (Boyar et al., 2001).

As in our patient, anxiety, emotional lability, impulsivity, and aggressiveness have been described as major behavioural problems (Thomas et al., 1999). Recurrent psychosis has been linked to partial trisomy 15q 11q13 (Michelson et al., 2011).

Major physiognomic features, such as characteristic facial features or short stature, are often associated with interstitial duplication of $15 q 11 q 13$, but are more frequently associated with idic15 (Chamberlain and Lalande, 2010). Our patient presented with some facial dysmorphic features that are reported in more than $75 \%$ of patients (Battaglia, 2008).

Epilepsy is a common feature in this syndrome. Although its presentation in $15 q$ duplication disorder is more severe in individuals with idic15, our patient's epilepsy, which was initially easy to treat, evolved into a refractory epileptic syndrome. His seizure pattern with several seizure forms, predominantly tonic and atonic seizures, related to his EEG features and intellectual disability, is suggestive of Lennox-Gastaut syndrome, in this case a late-onset form.

Lennox-Gastaut syndrome belongs to a group of severe epileptic encephalopathies. Symptomatic cases are usually due to perinatal asphyxia, tuberous sclerosis, meningoencephalitis sequelae, cortical dysplasia, cranial trauma, and more rarely, tumours and metabolic dysfunction. Chromosomopathies are rarely associated with this syndrome, although $15 q$ deletions in AS may be an aetiological background for such a syndrome (Campos-Castelló, 2004).

For some years, the patient's history of resuscitation at birth may have lead to the conclusion that his developmental delay was due to hypoxic-ischaemic complications. However, MR imaging studies did not confirm the existence of ischaemic lesions or other structural anomalies, making the diagnosis of symptomatic LGS due to $15 q$ duplication more plausible.

Although largely described with several forms of epileptic seizures, to our knowledge there is so far only one report of a LGS patient with a $15 q$ interstitial duplication. This was also a case of late-onset LGS associated with a microduplication of 15q11-q13 (Orrico et al., 2009). Four other cases of LGS were reported, but in idic15 patients (Battaglia, 2008).

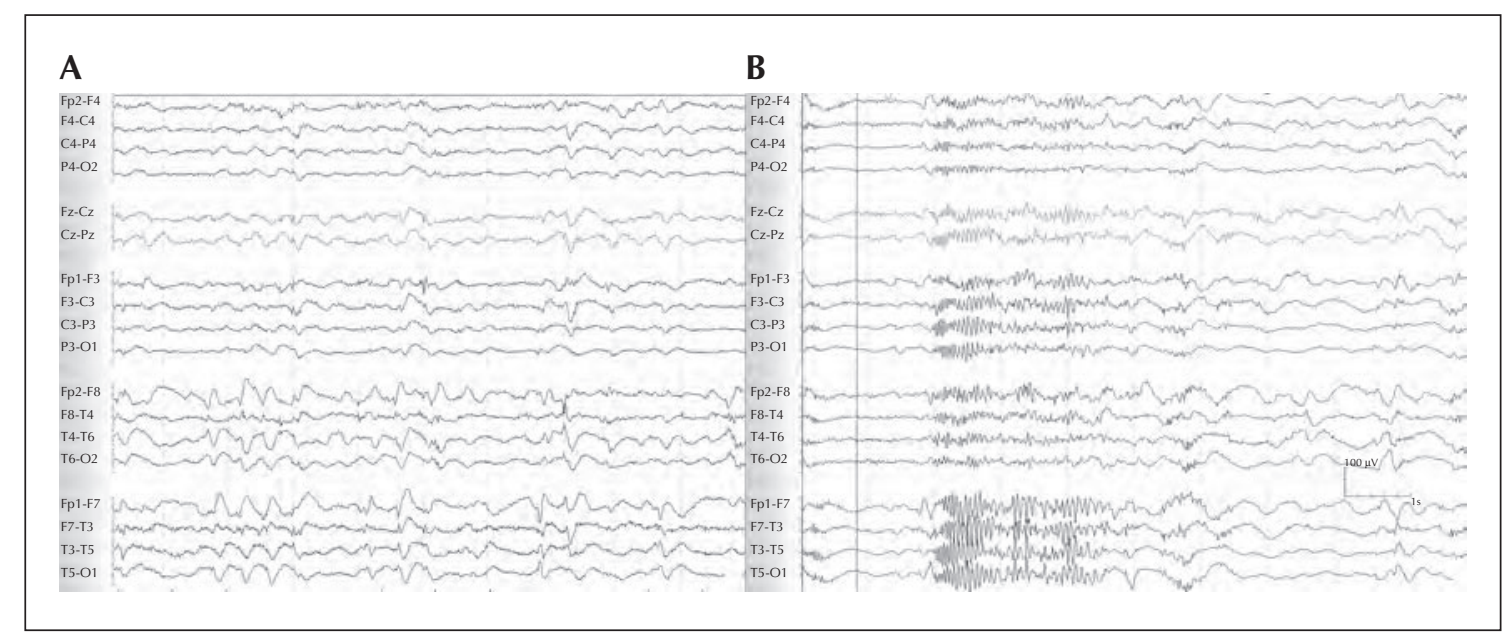

Figure 2. EEG: (A) generalised slow spike-wave complexes during drowsiness. (B) generalised fast paroxysmal activity in sleep. 
Developmental delay, present since early childhood, progressed to profound intellectual disability with increasing aggravation. This worsening coincided with the emergence of epilepsy, with progressive changes in seizure type and evolution of EEG recordings from normal to that of a generalised epileptic syndrome. In our patient, such as in the previous reported case of late-onset LGS, cognitive deterioration was probably symptomatic to the epileptic encephalopathy (Orrico et al., 2009).

As there is scarcity of information concerning clinical outcomes in adults, continuous reports of follow-up of $15 q$ duplication patients is encouraged to better characterise the adult phenotype, since most cases are paediatric.

\section{Disclosures.}

None of the authors has any conflict of interest to disclose. There was no financial funding for this report.

\section{References}

Battaglia A. The inv dup(15) or idic(15) syndrome: a clinically recognizable neurogenetic disorder. Brain Dev 2005; 27: 365-9.

Battaglia A. The inv dup (15) or idic (15) syndrome (tetrasomy 15q). Orphanet J Rare Dis 2008; 3: 30.
Battaglia A, Parrini B, Tancredi R. The behavioral phenotype of the idic(15) syndrome. Am J Med Genet C Semin Med Genet 2010; 154C: 448-55.

Boyar FZ, Whitney MM, Lossie AC, et al. A family with a grandmaternally derived interstitial duplication of proximal $15 q$. Clin Genet 2001; 60: 421-30.

Campos-Castelló J. Lennox-Gastaut syndrome. In: Orphanet Encyclopedia. September 2004. http://www.orpha.net/data/ patho/GB/uk-Lennox.pdf.

Chamberlain SJ, Lalande M. Neurodevelopmental disorders involving genomic imprinting at human chromosome 15q11q13. Neurobiol Dis 2010; 39: 13-20.

Cook Jr EH, Courchesne RY, Cox NJ, et al. Linkagedisequilibrium mapping of autistic disorder, with 15q11-13 markers. Am J Hum Genet 1998; 62: 1077-83.

Michelson M, Eden A, Vinkler C, et al. Familial partial trisomy $15 q 11-13$ presenting as intractable epilepsy in the child and schizophrenia in the mother. Eur J Paediatr Neurol 2011; 15: 230-3.

Orrico A, Zollino M, Galli L, Buoni S, Marangi G, Sorrentino $\mathrm{V}$. Late-onset Lennox-Gastaut syndrome in a patient with 15q11.2-q13.1 duplication. Am J Med Genet A 2009;149A: 1033-5.

Thomas NS, Browne CE, Oley C, Healey S, Crolla JA. Investigation of a cryptic interstitial duplication involving the Prader-Willi/Angelman syndrome critical region. Hum Genet 1999; 105: 384-7. 